Ann. Génét. Sél. anim., I979, 11 (2), I33-I43.

\title{
Origine du Mouflon de Corse dans une nouvelle perspective paléontologique : par marronnage
}

\author{
F. POPLIN \\ Laboratoive d'Anatomie compavée du Muséum national d'Histoire naturelle, \\ 55, rue de Buffon, 75005 Paris.
}

\begin{abstract}
Résumé
Le cheminement paléontologique du Mou flon de Corse est retracé à l'intention des généticiens afin de leur proposer une estimation du degré de parenté avec les ovins sauvages et domestiques actuels. Il ne s'agit pas d'une simple compilation bibliographique, mais d'une révision, peu d'attention ayant été accordée à la question jusque-là.

Contrairement à l'opinion générale, le Mou flon de Corse ne constitue pas, avez celui de Sardaigne, une espèce sauvage indigène. Il est né du marronnage de moutons encore primitifs introduits par l'Homme dès le début du Néolithique (6e, peut-être $7^{\mathrm{e}}$ millénaire). Le type originel sauvage d'Asie Mineure n'a pas été retrouvé exactement en raison d'une dérive génique due à l'isolement et au petit nombre de sujets ayant fait souche. Le Mouflon de Sardaigne a connu une évolution semblable, un peu plus infléchie vers les formes domestiques (femelles acères) parce que les croisements avec elles ont été plus faciles qu'en Corse. 11 n'est pas douteux que le Mouflon de Corse et de Sardaigne, celui du Proche-Orient (au moins) et les diverses formes de moutons domestiques restent conspécifiques et ne se distinguent qu'au niveau de la race ou de la sousespèce, dans des proportions que la Génétique devrait pouvoir préciser.
\end{abstract}

\section{Avant-propos}

A la source de ces pages, il y a un colloque organisé par la Société d'Ethnozootechnie à 1'École Nationale Vétérinaire d'Alfort sur les débuts de l'élevage du Mouton pour laquelle il m'avait été demandé de retracer l'aspect paléontologique (PoPlIN, I977). Au cours de cet exercice, je me suis aperçu avec un peu d'étonnement que le Mouflon de Corse avait été déclaré comme sauvage et indigène par d'anciens auteurs, et qu'on n'était pas revenu sur cette opinion fondée plus sur la conviction que sur l'observation de faits positifs.

Ayant exprimé, mais succinctement, un avis contraire qui a déjà été repris par certains auteurs comme BuNCH et al. (1978), il a paru utile de le développer pour en faire connaître les documents et 1'argumentation. Ce qui suit expose l'essentie1 
et renvoie pour le reste à une bibliographie abondante mais sélectionnée, qui assure l'intégration dans la connaissance et la problématique générales des Ovinés actuels et fossiles. A noter que tout cela traite davantage de Paléontologie, de Zoologie que de Zootechnie : mon propos est de soumettre aux généticiens, qui, eux, peuvent recourir à l'expérimentation biologique, une hypothèse de travail fondée sur les restes irrévocables du passé.

\section{Introduction}

Classiquement, les ouvrages traitant du Mouflon de Corse en font un animal sauvage installé depuis la nuit des temps sur les hautes pentes de 1'Ile de Beáuté - ou bien ils évitent d'aborder la question de ses origines. Le plus important 1ravail français sur les grands Mammifères des îles méditerranéennes, celui de VAUFREY (I929), indique que le Mouflon est connu comme fossile en Corse et qu'il " faisait assurément partie de la faune pléistocène, car c'est aujourd'hui (...) une espèce relique qui n'existe plus qu'en Corse et Sardaigne et en Crête " (1). VAufrey se fondait d'une part sur des ossements, ou plutôt sur un ossement trouvé au siècle dernier, auquel je reviendrai bientôt, d'autre part sur l'argument qui vient d'être cité. Celui-ci n'est pas solide. Il part de la conclusion, laquelle n'est qu'une supposition. Ce qu'il s'agirait de démontrer d'abord, ce serait la présence de restes pléistocènes dans les couches géologiques.

\section{Le "Mouflon fossile » de Corse}

VAUFREY fait appel aux recherches de LOCARD (I872) dans les brèches de Bastia, qui fit déterminer ses pièces par LOR'TET, alors Directeur du Muséum de Lyon. Il n'est pas superflu de citer le passage de LOCARD consacré au Mouflon : "Ovis musimon, LrNnÉ. - (Rare). Mouflon, ou probablement une autre espèce semblable; côte ".

Dans le même matériel, il y avait des indices de la présence de 1'Homme; il s'agissait de Néolithique. La première précaution à prendre était de penser à un mouton, voire à une chèvre. Mais ce réflexe salutaire n'était sans doute pas acquis en I872. Il est plus regrettable que VAUFREY ne 1'ait pas eu $\left({ }^{2}\right)$. J'ai fait demander au Muséum de Lyon si cette fameuse côte existait toujours, pour vérification anatomique et surtout dans l'espoir d'une datation CI4; elle n'a pas été retrouvée.

Voilà sur quoi reposait la théorie du Mouflon pléistocène en Corse. Dans l'état actuel des connaissances, il n'y est toujours pas attesté $\left({ }^{3}\right)$.

(1) Indépendamment du fait qu'il confond Chypre et la Crête, VAUfrey assimile dans cette page Ovis musimon de Corse et Sardaigne et Ovis ophion de Chypre. En soi, ce n'est pas un tort, mais il le fait en conservant les deux binômes, ce qui n'est pas logique. C'est pour éviter cette sorte d'ecueil que j'ai choisi de ne pas recourir à la terminologie latine. La nomenclature des ovins est très versatile; elle apporterait ici plus de confusion que de clarté, comme souvent quand se rencontrent Paléontologie, Zoologie et Zootechnie.

(2) Dans le même matériel, le Renard est aussi mal représenté ("Côtes "). VAưfrey ne manque pas de le souligner. Mais s'il s'exclame sur la valeur dérisoire de ces côtes de Renard, il est plein de complaisance pour celle du "Mouflon ". Ce favoritisme allait, bien sûr, dans le sens de sa conviction.

(3) Mon collègue Gasc, à l'occasion de son Diplôme d'Études Supérieures, terminé en I961 et resté inédit (voir cependant GASc, 1969), s'est posé la question dn Mouffion à propos d'un talus ( $=$ astragale) de Macinaggio, concluant plutôt à un Cervidé. I a pièce me paraît en effet appartenir à ce groupe. 
Pour un tel problème, les enseignements généraux de la Paléontologie dictent le schéma de principe suivant : on devrait voir se constituer en Corse à un moment plus ou moins éloigné dans le temps une population détachée de la vaste nappe du genre Ovis, à partir d'une région continentale proche. Puis on devrait assister à une différenciation, la population isolée évoluant pour son propre compte et pouvant donner une race, une sous-espèce, une espèce, voire un genre propre ou plus encore. On connaît dans les îles méditerranéennes un certain nombre d'animaux très particuliers, ayant évolué un long temps en vase clos, qui constituent des reliques $\left({ }^{1}\right)$. Même quand on ne dispose pas de la lignée de leurs ancêtres, on est fondé à y voir les effets d'une longue ségrégation. C'est une manière de situer le débat au plan de l'Anatomie comparée actuelle qui est à la fois le complément et le corollaire de la réflexion sur les fossiles, et le moment viendra plus loin de se demander si, de nos jours, le Mouflon de Corse tranche sur les autres ovins. Dans l'immédiat, il convient d'en terminer avec les formes anciennes.

\section{Les Mouflons fossiles du Continent}

Nous avons vu qu'il n'y en a pas de véritable en Corse. Supposons que ce soit seulement parce qu'il n'en a pas été trouvé, et tournons-nous vers le Continent proche pour voir s'il n'y a pas existé des mouflons durant le Pléistocène. Actuellement, les ovins sauvages s'étendent de 1'Asie Mineure à 1'Extrême-Orient et audelà du détroit de Behring jusque dans les Montagnes Rocheuses. Ils sont foncièrement orientaux; d'ailleurs, le plus ancien fossile connu pour être sûrement un Mouflon, Ovis shantungensis du Nihowan, vieux de plus d'un million d'années, a été trouvé en Chine (TeIlmard de Chardin et Piveteau, r930). A partir du foyer asiatique, l'aire de répartition s'est étendue au cours du Pléistocène vers l'Est à travers toute l'Amérique du Nord (Ovis catclawensis, par exemple) où les restes abondent. Vers 1'Ouest, il y eut de rares incursions en Europe au Pléistocène moyen, c'est-à-dire à une époque qui remonte à plusieurs centaines de milliers d'années. Des jalons se trouvent en Moravie et en France. En Moravie, 1a détermination d'Ovis faite par NEHRING (I89I) n'a toujours pas été mise en doute sérieusement (PAyne, I968); elle est d'ailleurs confirmée par MusiL (Ig68 et in litt.), et cet auteur a ajouté en I 968 de fort belles pièces provenant de la région de Brno. En France, la remarquable découverte faite par Pommerol (I88o, I882) à Pontdu-Château, en Auvergne, vient d'être suivie de deux autres à Sauveterre-laLémance, dans le Lot-et-Garonne (DELPECH et al., I978) et à Tautavel, dans les Pyrénées-Orientales (CRÉGUT, I979). Il m'a été donné de participer de près aux identifications, dans les deux derniers cas. Grâce au matériel de comparaison dont dispose le Muséum national d'Histoire naturelle, il ne subsiste aucun dcute sur l'attribution générique de ces restes. Pour le Mouflon de Pont-du-Château, il est à craindre que le matériel ne soit définitivement perdu. Il semble avoir été conservé au Musée de Clermont-Ferrand puis au Laboratoire de Géologie de l'Université de cette même ville. Les multiples démarches que j'ai faites sont malheureusement restées vaines. Cependant, grâce aux figurations que donne POMMEROL, et malgré quelques maladresses de description de la part de cet auteur (notamment

(1) En Corse, le petit Lagomorphe Prolagus, le Canidé Cynotherium en sont des exemples, même s'ils ne sont pas parvenus jusqu'à nous. 
en ce qui concerne la fosse larmière), la certitude d'un Ovis est acquise (1).

Ces différents restes conduisent à deux constatations : il se situent en Europe du Sud, dans 1'Europe des montagnes comme il fallait s'y attendre, et sont de très grande taille, égale ou même supérieure à celle du Mouflon de Marco Polo et de l'Argali, qui sont les plus grands ovins sauvages actuels. C'étaient des animaux grands comme un cerf de France. Auprès d'eux, le Mouflon de Corse est un nain. Bien sûr, une conséquence de l'insularité étant, pour les grandes espèces, le rapetissement (et au contraire le gigantisme pour les souris et autres micro-mammifères), on pourrait penser qu'en quelques centaines de milliers d'années, la stature a pu diminuer ainsi. Mais il serait pour le moins heureux en ce cas qu'une suite de fossiles corses montrât cette involution. Or, il n'y en a pas. Si l'on s'en tient à ce dont on dispose, il faut bien constater que le Mouflon continental actuel le plus proche en grosseur est celui du Proche-Orient - et c'est aussi le plus proche dans l'espace.

Dans tout cela, il est à souligner qu'il n'a pas été trouvé de ces vieux et grands mouflons fossiles dans la péninsule italienne, notamment pas en Toscane, en face de la Corse, là où se situerait le mieux le point de départ de la traversée de la Mer Tyrrhénienne $\left({ }^{2}\right)$.

Le Mouflon ne s'est pas maintenu longtemps en Europe. Il y a plutôt fait des incursions (comme un Buffle asiatique à peu près à la même époque, trouvé à Steinheim, en Allemagne) rares et anciennes. En particulier, les rivages de la Méditerranée occidentale n'en ont plus revu. Cela a pourtant été avancé par quelques auteurs, comme BOULE (I929) dont la notoriété a eu un rôle malheureux. Les ossements d'époque pléistocène récente qu'il a attribués à un ovin sauvage sont soit de Chamois (grottes de Lestellas et de Montardit, en Ariège), soit de Mouton (domestique) qu'il prenait pour plus anciens qu'ils n'étaient. A sa suite, d'assez nombreuses erreurs du même genre ont été commises, mais ce n'est pas ici le lieu d'en entreprendre la revue, et il me déplairait de mettre en cause des contemporains. Qu'il suffise de dire qu'en l'état actuel des connaissances, la présence chez nous d'un ovin précédant le Mouton domestique n'a rien de sûr, et qu'il faudrait, pour la démontrer, des datations $\mathrm{Cr}_{4}$ significatives faites sur les os mêmes, et que ceux-ci soient des pièces ne laissant pas de doute quant à leur appartenance au genre Ovis.

La domestication du Mouton a commencé dans les régions centrales du ProcheOrient et Moyen-Orient, sur le Mouflon local au cours du $8^{\mathrm{e}}$ millénaire $\left({ }^{3}\right)$. Des dates plus anciennes ont été avancées, surtout par PER KINs (I964), mais sur des bases méthodologiquement déficientes; l'enthousiasme de la recherche " du plus vieux mouton du monde " me paraît avoir eu une part trop belle dans l'affaire. Il faut dire qu'entre Mouton primitif et son ancêtre direct, il y a fort peu de diffé-

(1) Un fragment crânien trouvé à Sénèze serait aussi de Mouflon, et plus ancien que ces restes du Pléistocène moyen (SCHAUB, 1923, 1937, 1944). Mais la pièce est difficile à identifier (il est regrettable que SchaUb n'en reparle pas quand il crée son Soergelia en I95I), et d'origine stratigraphique incertaine. Aussi préférai-je en faire abstraction pour m'en tenir aux choses sûres. En Angleterre, Caprovis savini du Cromérien ne me paraît pas un vrai ovin. En Allemagne, les déterminations anciennes d'Ovis (par Freudenberg en rgr4 notamment) sont fondées sur une méprise (=Ammotragus considéré comme un ovin), et/ou concernent des pièces très fragmentaires, comme à Mosbach.

${ }^{2}$ ) Il est à peine besoin d'indiquer que le Mouflon de Corse ne saurait dériver du Mouflon à manchettes d'Afrique du Nord (Ammotragus levvia). Cet animal, qui a trop longtemps été pris pour un ovin, est en fait un caprin. C'est en quelque sorte la forme de Bouquetin propre à l'Afrique blanche.

(3) I a classification des Mouflons de ces régions étant mouvante, j'envisage ici et dans la suite l'ensemcle des populations qui s'étendent du Bosphore à la Perse comprise, ce qui correspond au regroupement d'O. orientalis et d'O. vignei (sauf Chypre) dans BuNCh et FoOTe (1977) qui suivent Gerst (I97I), d'O. ammon gmelini et d'O. ammon orientalis dans BANNIkov et HEPTNER (1972) qui suivent là-dessus PFFFFER (1967), d'O. musimon continental et d'O. orientalis dans BököNYI (1964), d'O. orientalis (sauf Chypre et peut-être Punjab) et d'O. laristanica dans Fllermann et Morrison-ScotT (I95I). 
rence ostéologique, et que le risque de placer la limite trop loin dans le temps était maximale dans la région habitée par cet ancêtre - c'est là que se situent les recherches en question. D'autre part, le Mouflon ne s'est pas trouvé du jour au lendemain installé en bergerie, couvert de laine et sélectionné. Il y eut une gradation qui a duré des siècles et des millénaires. Il faudrait s'entendre sur "le début " de la domestication. Sur ce chapitre, on observe depuis quelques années une tendance à croire que la chasse était un état d'exploitation anarchique, proche du chaos primitif, et que dès que l'on observe une régularité dans l'exploitation des animaux (on. veut dire par là : dans l'abattage, c'est-à-dire dans leur courbe de mortalité), c'est que l'élevage, ou un certain mode de protoélevage avait commencé. Mais c'est une grande injure faite aux peuples chasseurs préhistoriques que de ne pas leur supposer la faculté d'organiser leur prédation. La prévoyance fait partie de l'intelligence de la chasse, et elle n'a pas attendu l'homme du $\mathrm{xx}^{\mathrm{e}}$ siècle pour se développer. Tout cela pour dire qu'il ne faudrait pas tirer prétexte de toute systématisation de l'exploitation du stock animal pour apposer l'étiquette de domestication, sinon la porte serait ouverte aux contresens, et on risquerait de faire reri. onter l'élevage loin dans le Pléistocène. Il vaut mieux en rester à la bonne vieille définition d'un état de dépendance où l'homme contrôle la vie des bêtes reproduction comprise. Le lecteur généticien, du moins, acquiescera, puisque derrière le contrôle de la reproduction, il y a la notion de sélection artificielle.

Donc, le Mouton est né en Asie Mineure il y a 9 ou ro ooo ans, et a diffusé en Europe : il l'aborde au milieu du $7^{\circ}$ millénaire, et parvient quelques siècles plus tard en France du Sud, le long de son littoral méditerranéen, alors que sa pénétration en France du Centre et du Nord sera nettement plus tardive. Tout semble se passer comme si les premiers troupeaux avaient suivi le bord de mer, et non pas les voies transcontinentales. Or, ils parviennent aussi bien en Corse : les fouilles de BAIlloud à Basi (première moitié du $6^{\mathrm{e}}$ millénaire) semblent l'indiquer, quoique l'étude de la faune ne soit pas achevée. Il a fallu traverser à l'époque une Mer Tyrrhénienne qui différait peu de l'actuelle. Par conséquent, la propagation du Mouton dans le bassin de la Méditerranée occidentale semble au moins autant une affaire de navigation et de cabotage que de marche à pied.

Il apparaît de plus en plus clairement, à l'échelle de la Méditerranée toute entière, que la navigation a précédé l'élevage du Mouton, et que celui-ci a précédé la céramique. Celle-ci existe déjà à Basi. On sait d'autre part, grâce aux fouilles de LANFRANCHI et WEISS, que 1'Homme avait déjà atteint la Corse au milieu du $7^{\mathrm{e}}$ millénaire. On peut s'attendre à y trouver le Mouton dès 6000 avant notre ère.

En tenant compte des incertitudes et des divergences d'opinions, on peut dire que le Mouton aura mis un ou deux millénaires pour venir d'Asie Mineure en Corse. C'est peu, en comparaison des 9 ou ro mille ans de son histoire. Il devait être encore très primitif. Par exemple, ce n'est que bien plus tard que sa toison deviendra laineuse (RYDER, I973, I974). On peut donc penser que des sujets ayant échappé à la surveillance de l'Homme ont pu facilement constituer une population marronne, qui n'avait pas un grand chemin paléontologique/génétique à rebrousser pour constituer ce que nous appelons le Mouflon de Corse.

Il faut souligner à cet égard la prédisposition écologique de l'île : elle offre les mêmes paysages que les régions montagneuses du Proche-Orient, avec le même type de végétation et de climat. Et les sommets constituent des refuges contre le contrôle de l'Homme; mais ce rôle de défense farouche des paysages corses est trop proverbial pour qu'il y ait besoin d'y insister.

Cela vaut pourtant la peine en le faisant de manière comparative avec la Sardaigne, très proche, où existent également des mouflons, assurément de même 
origine. Cette île est moins accidentée que la Corse, et les mouflons y ont un type sauvage moins marqué : les femelles sont souvent dépourvues de cornes. Il semblerait que les refuges moins bons aient moins protégé contre les croisements avec les moutons domestiques.

Une autre comparaison mérite d'être faite en Corse même, avec les Suidés, probablement introduits eux aussi par 1'Homme. Entre les cochons domestiques traditionnels et leurs confrères sauvages ( $=$ descendants de sujets marrons eux aussi, sans doute), il y a bien peu de différence. Il faut dire que l'occasion de se croiser leur est largement octroyée, puisque les porcs sont lâchés en pleine liberté dans la nature plusieurs mois de l'année. Les pratiques des bergers sont plus strictes. Mais surtout, comme le Sanglier descend beaucoup plus bas que le Mouflon, il lui est beaucoup plus facile de mêler ses gènes à ceux de ces congénères domestiques.

Tout cela encourage à voir dans les mouflons de Corse et de Sardaigne $\left({ }^{1}\right)$ des produits du marronnage, les premiers ayant réussi mieux que les seconds à rester isolés des moutons domestiques. Comment ces derniers ont-ils évolué? I1 faut bien avouer que les études manquent encore pour donner une ébauche détaillée de leur histoire, bien que les recherches en Corse aient commencé il y a plus d'un siècle et demi (Cuvier, I823). Toutefois on trouve dans cette race ovine de Corse des caractéristiques relativement archaïques comparées à celles des ovins continentaux aussi bien pour la laine et la conformation (QUIT'TE'T, I965) que pour la coloration de la toison (LAUVERGNe et ADALSTEINSSON, I976). On peutdonc penser que le Mouton corse sans échapper au flux de gènes qui ont abordé 1'Europe occidentale a présenté aux cours des siècles une certaine réticence aussi bien pour accepter les nouveaux gènes que pour rejeter les anciens, ce qui selon $\mathrm{L}_{4} \mathrm{AU}-$ VERGNE (I979) en ferait un modèle de race archaïque en Méditerranée occidentale.

En résumé, les restes fossiles indiquent qu'il n'y a pas eu d'ovin en Corse avant l'arrivée de l'Homme et de l'élevage, à 1'Holocène, et que s'il y a bien eu des mouflons en Europe continentale au Pléistocène moyen, il y a plusieurs centaines de milliers d'années, ce fut sans liaison avec l'animal insulaire que nous connaissons. Certains auteurs avancent l'hypothèse d'une migration par terre ferme à cette époque ancienne. Si elle avait eu lieu, la Corse aurait hérité du même coup des chevaux, bisons, ours, hyènes etc... qui n'auraient pas manqué de suivre le mouvement, et sur 1'absence desquels l'accent ne saurait être trop mis.

\section{Introduction de moutons primitifs ou de gibier?}

Le résultat atteint à ce point de l'exposé est que le Mouflon n'est pas arrivé en Corse avant l'Homme et la domestication. Mais a-t-il connu celle-ci, fut-ce brièvement? On pourrait suggérer qu'il ait été introduit comme gibier, par exem-

(1) L'introduction par l'Homme a déjà été avancée par BACETTI (1964), mais pour la Sardaigne seulement, et sans grande argumentation. L'auteur ne fait pas expressément mention d'un passage par uv début de domestication, c'est-è-dire qu'il ne formule pas la thèse du marronnage. D'autre part, il n'argumente guère sur l'absence d'ovin pléistocène en Sardaigne, alors que Lovisaro (in: IsSEL, I9I4) en a noté dans le Sud de l'île, ce qu'ont relevé Joleaud (r926) puis Vaufrex (I929). Il s'agissait en fait de restes très mal conservés ("in pessimo stato di conservazione ", sur lesquels aucune précision n'est donnée, de sorte qu'on peut douter de la validité de l'observation. Plus récemment, MaLATESTa (i97o) a signalé le Mouflon avec réserve ("? Ovis musimon Pallas (juv. )) dans la grotte de Dragonara; mais il a eu l'obligeance de me signaler (in litt.) qu'il ne s'agit pas de Mouflon, ajoutant qu'il ne croit pas lui non plus à un Ovis pléistocène das la région Corse-Sardaigne. 
ple par les Romains qui ont su peupler plusieurs îles de Méditerranée en Cervidés $\left.\mathbf{(}^{\mathbf{1}}\right)$. Mais ceux-ci se trouvaient à proximité, comme en Italie, alors que les mouflons étaient à l'autre extrémité de la Méditerranée orientale. Pour cette raison, 1'hypothèse du marronnage est la plus vraisemblable, dans l'état des connaissances paléontologiques et paléobiogéographiques.

\section{Parenté actuelle du Mouflon de Corse}

Il est temps, maintenant qu'ont été examinés les liens possibles à travers le temps et l'espace, d'en venir au degré de parenté avec les ovins actuels, sauvages et domestiques. L'expérience de la Paléontologie des grands Mammifères amène à penser qu'un isolement depuis le Pléistocène moyen aurait produit au moins une espèce nouvelle, alors qu'une ségrégation ne remontant pas à ro 000 ans n'aurait pu donner qu'une race, une sous-espèce tout au plus. Or nous savons que le Mouflon de Corse (et Sardaigne) est largement interfécond avec le Mouton domestique, ainsi qu'avec le Mouflon d'Asie Mineure, pour ne parler que de lui. Il est maintenant évident que lorsqu'on a donné, jadis, un statut spécifique à $O$. musimon, ce fut en faisant implicitement le pari que son insularité assurait son identité et le garantissait de droit de toute conspécificité avec ses congénères. Le pari a été perdu.

Cette étroite parenté avec le Mouflon proche et moyen-oriental et le Mouton domestique corrobore la thèse du marronnage, au détriment de celle de l'origine ancienne. D'autres données biologiques viennent l'appuyer, mais elles ne sont pas du ressort du spécialiste des ossements fossiles. Je n'en retiendrai qu'une, celle mise en évidence par Bunch et al. (I978) sur la constitution des hémoglobines. Ces auteurs ont montré que le Mouflon de Corse (et Sardaigne) partage avec le Mouton le type $A$, que ne possèdent pas les autres Mouflons actuels.

En conclusion, le Mouflon de Corse peut être considéré comme un Mouton manqué, relativement proche des moutons traditionnels de la même île, assez distant probablement des moutons continentaux (en particulier des races modernes très sélectionnées). On peut présumer que le Mouflon de Sardaigne lui est particulièrement proche, que les ovins "sauvages " et domestiques traditionnels des deux îles, s'ils ne constituent pas un véritable ensemble, forment tout au moins une communauté génétique diffuse, se situant, du point de vue évolutif, entre le Mouflon du Proche et Moyen-Orient et les races domestiques modernes. Il est à souhaiter que 1'expérimentation génétique précise ces " distances", et que les recherches sérologiques livrent les relations d'ordre supérieur, comme cela est fait maintenant pour les Primates.

Reçu pour publication en mai 1979.

\section{Remerciements}

Ils vont à M. Lauvergne qui m'a suggéré de faire cet article et l'a relu, ainsi qu'à M. PfefFER dont l'encouragement et le conseil m'ont été précieux. Ils vont aussi au Prof. Malatesta pour ses renseignements sur le matériel de Dragonara, et à Mme Mouré pour sa recherche des pièces de Locard au Muséum de Lyon.

(') Certains auteurs pensent, non sans raison, que le Cerf a été introduit en Corse. D'autre part, il est curieux qu'on n'y ait pas trouvé de restes de Daim dans les périodes historiques. 


\title{
Summary
}

\author{
The Origin of the Corsican Mouflon \\ in a new paleontologic prospect: by teralization
}

The paleontological course of the Corsican Mouflon is retraced here in the interests of geneticists, so as to provide them with an estimate of the degree of affinity between these animals and the present-day members of the sheep family, both wild and domestic. It is not merely a matter of compiling a bibliography but one of revision, for little attention has been paid to the question up to now.

Contrary to general opinion, the Corsican Mouflon, along with that of Sardinia, is not an indigenous wild species. It originates with the Sheep, introduced by Man while still at a primitive stage, right at the beginning of the Neolithic (6th or fossibly 7 th millenium), which reverted to the wild. The original wild type from Asia Minor is not reproduced exactly because of a genetic drift resulting from isolation and from the limited number of individuals which have successfully reproduced. The Sardinian Mouflon has undergone a similar evolution, though its modification tends slightly more towards the domestic forms (hornless females), since interbreeding was easier than in Corsica. There is no doubt that the Corsican and Sardinian Mouflon, like that of (at least) Near East and the various forms of domestic Sheep, belong to the same species, being distin€uished only at the racial or sub-specific level, in proportions which it $\leqslant$ hould be possible to make clear through genetics.

\section{Références bibliographiques}

BAcettr B., 1964. Considerazione sulla costituzione e l'origine della fauna di Sardegna, in: Problemi di Biogeografia della Sardegna, Lav. Io Cong. Soc. Itat. Biogeog. Sassari I964, Arch. Botanico e Biogeografico Italiano, 40, $4^{\mathrm{e}}$ sér., 9 (= Atti Soc. Ital. Biogeog. 1963-64), 2 I 7-283, 6I fig.

Banni kov A. G. et Heptrer (W. G.), 1972. (Les Mouflons autres que celui de Corse et Sardaigne) in: Grzime K (B.) et Fontaine (M.). Le monde animal en I3 volumes, 13, 462.

Boessneck J., Müller H. H. et Teichert M., r964. Osteologische Unterscheidungsmerkmale zwischen Schaf (Ovis aries LrNNe) und Ziege (Capra hircus LinNe), Kühn-Archiv, 78, I/2, 129 p., 8o fig., Berlin.

BököNYI S., 1974. History of domestic mammals in central and eastern Europe, 597 p., $\mathbf{I}_{4} 8$ fig., nombreux tableaux, Budapest, Acad. Sc. Hong.

BöкöNyr S., 1977. The introduction of sheep-breeding to Europe, in: les débuts de l'élevage du Mouton, Colloque d'Ethnozootechnie, Alfort 1977 (Ethnozootechnie no 2I), Paris 1977, 65-70.

Bouchud J., 1955. Deux espèces rares au Moustérien découvertes au Pech de l'Azé. Bull. Soc. Préhist. Fr., 52, 89-93, I pl.

Bouchud J., 1956. La faune Epimagdalénienne et Romanello-Azilienne en Dauphiné. Bull. Mus. Anthrop. Préhisi. Monaco, 3, 177-1 87 .

Bouchud J., 1956. La faune de l'abri Pagès, in: Nrederlender A., I.acam R. et SonnevilleBordes (D. de). L'abri Pagès à Rocamadour et la question de l'Azilien dans le Lot. L'Anthropologie, 60, 444-446.

Bouchud J., 1959. La petite faune de la grotte du Lazaret, locus $8,2^{\text {e }}$ note. Bull. Mus. Anthrop. Préhist. Monaco.

Bouchud J., 1973. La grosse faune et les oiseaux, in: Bouchud J. et Desbrosse R. La faune de la grotte des Freydières à Saint-Agnan-en-Vercors (Drôme). Bull. Soc. Préhist. Fr., 70, 330-334, I fig., 6 tabl.

Boule M., 1927. Ovis sp., in : Boule M. et Villeneuve (L. de). La grotte de l'observatoire à Monaco. Arch. Inst. Paléont. Humaine, Mém. I, Paris, Masson, 45-50, fig. 9, pl. 4.

Boule M. et Teilhard de Chardin P., i928. Paléontologie, in : Boule M., Breuil H., licent E. et Terlhard P. Le Paléolithique de la Chine. Arch. Inst. Paléont. Humaine, Mém. 4, Paris, Masson 27-102, fig. I2-29. 
Brink F. H. van den et Barruel P., I967. Guide des Mammifères d'Europe, 260 p., 32 pl., 135 cartes, Delachaux et Niestlé, Neuchâtel.

Bunch T. D. et Foote W. C., 1977. Cytogenetic evidence on the ancestral stock of domestic sheep (Ovis aries), in: les débuts de l'élevage du mouton, Colloque d'Ethnozootechnie, Alfort r977 (Ethnozootechnie no 2I), Paris I977, I1-I 5 .

Bunch T. D., N'Guyen T. C. et Lauvergne J.-J., 1978. Hemoglobins of the Corsico-Sardinian Mouflon (Ovis musimon) and their implications for the origin of $\mathrm{Hb} A$ in domestic sheep (Ovis aries). Ann. Génét. Sél. anim., 10, 503-506.

Camps G., I976. La question des navigations préhistoriques dans le bassin occidental de la Méditerranée. Cong. Préhist. de France, $20^{\mathrm{e}}$ sess., Provence, I974 (1976), 53-62, 5 fig.

CAmps G., I976. La navigation en Francc au Néclithique et à l'âge du Bronze, in : la préhistoire française, 2, sous la dir. de J. Guilaine, I92-20I, 4 fig. Paris, C.N.R.S. éd.

C.amps G., I977. Trois problèmes de la Préhistoire corse, in: SAUTUOLA 2, Santander, I75I 87 , I fig., 2 pl.,

Clark J. L., 1964. The great arc of the wild sheep, 247 p., nombreuses fig., Univ. Oklahoma Press, Norman.

Clutton-Brock J. et Uerpmann H.-P., 1974. The Sheep or early Jericho, J. Archeol. Sci, 1, 26I-274, 10 fig., 3 tabl.

Cornevin C. et Lesbre F. X., i 89r. Caractères ostéologiques différentiels de la Chèvre et du Mouton. Bull. Soc. Anthrop. Lyon, 10, 47-72.

Crégut E., 1979. La faune de Mammifères du Pléistocène moyen de la Caune de l'Arago à Tautavel (Pyrénées-Orientales), Thèse de $3^{\mathrm{e}}$ cycle, Université de Provence, $38 \mathrm{I}$ p., 57 fig., nombreuses planches, I 93 tabl.

Cuvier G., r823. Sur les os de Ruminans incrustés dans les brèches osseuses qui remplissent les fentes de rochers à Gibraltar et dans plusieurs autres lieux des cotes de la méditerranée, et sur ceux de quelques autres animaux qui les y accompagnent, in: Recherches sur les ossemens fossiles, $2^{\mathrm{e}}$ éd., 4, I98-203 : des brèches osseuses de Corse, 203-207: des brèches osseuses de Sardaigne, pl. I4-I 5 .

Cuvier G., I 845 . Ido, $4^{\mathrm{e}}$ éd., 6, 394-403 et 404-4I2, pl. I75-1 76.

Delpech F. et Heintz E., r976. Les Artiodactyles : Bovidés, in: La préhistoire française, 1, sous la dir. de H. de I.umley, C.N.R.S. Paris, 386-394 (le passage sur Ovis est de F. DELPECH).

Delpech F., Le Tensorer J. M., Pineda R. et Prat F., I978. Un nouveau gisement du Pléistocène moyen : Camp-de-Peyre à Sauveterre-la-Lémance (Lot-et-Garonne). C. R. Acad. Sc., Paris, 286, sér. D, I IOI-I Io3.

Ducos P., r977. Les débuts de l'élevage du Mouton au Proche-Orient, in : les débuts de l'élevage du Mouton, Colloque d'Ethnozootechnie, Alfort 1977 (Ethnozootechnie no 21), Paris I977, 33-37.

Ellerman J. R. et MorRison-Scott T. C. S., I95I. Checklist of palaearctic and indian Mammals, 810 p., I carte, Londres (British Museum).

Epstern H., I971. The origin of the domestic animals of Africa, 2, 7I9 p., 627 fig., New-York, Londres, Munich.

FreCh KOP S., I955. Sous-ordre des Ruminants ou Sélénodontes, in: Traité de Zoologie, sous la dir. de P. P. Grassé, 17, Mammifères : les Ordres, 568-693, fig. 557-718, Paris, Masson.

Freudenberg W., I9I4. Die Säugetiere des älteren Quartärs von Mitteleuropa, mit besonderer Berücksichtigung der Fauna von Hundsheim und Deutschaltenburg in Niederösterreich nebst Bemerkungen über verwandte Formen anderer Fundorte, Geol. Palaeontol. Abh. (nouv. sér.), 12, 4-5, 453-671, 69 fig., pl. 29-48, Iéna.

Furon R., I96I. Documents paléogéographiques pour servir à l'histoire du peuplement des îles méditerranéennes. Coll. Intern. C.N.R.S. no 94 , le peuplement des îles méditerranéennes ct le problème de l'insularité, Banyuls-sur-Mer I959 (Paris I961), I 8-27.

Gasc J. P., I96I. Etude d'une faune quaternaire de la Corse. Grotte de Macinaggio, Dipl. Et. Sup., Fac. Sc., Univ. Paris, $7^{6}$ p.

Gasc J. P., 1969. Étude paléontologique des petites espèces trouvées dans la grotte de Macinaggio, in: Livret-guide de l'excursion Corse, sous la dir. de F. Otrman, $8^{\mathrm{e}}$ Cong. INQUA, Paris $1969,73-80$, fig. 22-25.

Gauthrer A., I976. Essai de bibliographie géologique de la Corse. Bull. Soc. Sc. Hist. Nat. de la Corse (en trois parties); également : Corse d'hier et de demain no $10,79 \mathrm{p}$

Gerst V., 197I. Mountain sheep, a study in behavior and evolution, Univ. Chicago Press, Chicago, Londres, 49 fig., 89 pl., 77 tabl.

Ginsburg L., 1973. La faune, in: les fouilles de Mariana (Corse). Corsica, 32, i 2 p., 3 pl. 
Guilaine J., I976. Premiers bergers et paysans de l'Occident méditerranéen, 286 p., 57 fig., Mouton, Paris - La Haye.

Guilaine J., I977. Les débuts de l'élevage du Mouton en France, in: les débuts de l'élevage $\mathrm{du}$ Mouton. Colloque d'Ethnozootechnie, Alfort 1977 (Ethnozootechnie $n^{0}$ 2I), Paris 1977, 103I05, I fig.

Haltenorth T., I963. Klassification der Säugetiere, I (18) Artiodactyla, in: Handbuch der Zoologie, 8, $32^{\mathrm{e}}$ liv., I67 p., I6 pl.

Herre W. et Kesper K. D., 1953. Zur Verbreitung von Ovis ammon in Europa, Zool. Anz., 151, 204-209.

Herre W. et Röhrs M., 1973. Haustieve — zoologisch gesehn, $24^{\circ}$ p., 46 fig., Gustav Fischer, Stuttgart.

ISSEL A., I9I 4. Lembi fossiliferi quaternari e recenti osservati nella Sardegna meridionale dal

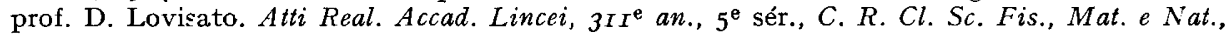
23, $759-770$.

Jeannel R., I96r. Le peuplement de la Corse et de la Sardaigne. Coll. Intern. C.N.R.S. $n^{\circ}$ 94. Le peuplement des îles méditerranéennes et le problème de l'insularité, Banyuls-sur-Mer I959 (Paris I96I), 35-39.

Joleaud L., 1926.Les Mammifères, in: Histoire du peuplement de la Corse. Bull. Soc. Sci. Nat. Hist. Corse (I ${ }^{\mathrm{er}}$ Mém. Soc. Biogéog.), 35-107.

Kurtín B., I968. Pleistocene Mammals of Europe, 3 I 7 p., i I I fig., 15 tabl., Londres, Weidenfeld et Nicolson.

LAUVERGNE J.-J., I 979. L'identité corse se vérifie aussi chez les animaux, Kyrn (93), 24-26.

Lauvergne J.-J. et Adalsteinsson S., 1976. Gènes pour la couleur de la toison de la Brebis corse. Ann. Génét. Sél. anim., 8 (2), I 53-1 72, 5 fig., 8 tabl.

Lauvergne J.-J., Denis B. et Thíret M., 1977. Hybridation entre un Mouflon de Corse (Ovis ammon musimon Schreber, I 872 ) et des brebis de divers génotypes; gènes pour la coloration pigmentaire. Ann. Génét. Sél. anim., 9 (2), I5I-I6r, I fig., 2 tabl.

Locard A., I 872 . Note sur les brèches osseuses des environs de Bastia (Corse). Arch. Hist. Nat. Lyon, 1, 37-5I.

Locard A., 1972-1973. Sur les brèches osseuses des environs de Bastia (Corse). Bull. Soc. Géol. France, $3^{\mathrm{e}}$ sér., 1, 232-234.

LYDEKKER R., I898. Wild oxen, sheep and goats of all lands, living and extinct, 318 p., 6I fig., $27 \mathrm{pl}$., Londres.

Malatesta A., 1970. Cynotherium sardous Studiati, an extinct Canid from the Pleistocene of Sardinia. Mem. Ist. Ital. di Pal. Umana, nouv. sér., 1, 72 p., 37 fig., Io pl.

Matsumoto H., I927. On a new fossil race of bighorn Sheep from Shantung. Sc. Reports of the Tohoku Imper. Univ. Sendai, 10, $2^{\mathrm{e}}$ sér. (Géologie), 39-4I, pl. I6-I7.

Musil R., I968. Neue Funde von Schafen in Mähren. Act. Musei Moraviae, 53, I63-176, I6 fig., $4, \mathrm{pl}$.

Nehring (A.), I89i. Diluviale Reste von Cuon, Ovis, Saiga, Ibex und Rupicapra aus Mähren. Neu. Jahrb. Mineral., Geol. Paleont., 2, I07-I55, 3 fig., 3 pl.

PAYNE S., 1968. The origins of domestic sheep and goats: a reconsideration in the light of the fossil evidence. Proc. Prehist. Soc., 34, 368-384, 2 tabl.

Perkins D., 1964. Prehistoric fauna from Shanidar, Iraq. Science, 144, I 565-66.

Perles C., 1979. Des navigateurs méditerranéens il y a ro ooo ans. La Recherche, 96, 82-83, 2 fig.

Petter F., 1973. Les animaux domestiques et leurs ancêtres, Paris, Bordas éd., i28 p.

Pfeffer P., 1967. Le Mouflon de Corse (Ovis ammon musimon Schreber, I782); position systématique, écologie et éthologie comparées. Mammalia, 31, suppl., 262 p., 35 fig., 6 pl., I carte.

Pilgrim G. E., I947. The evolution of the buffaloes, oxen, sheep and goats. Journ. Linn. Soc. London, 41, 272-286.

Piveteau J., I96r. Quelques Mammifères singuliers de Corse et des îles Baléares et leur signification paléogéographique. Coll. Intern. C.N.R.S. $n^{0}$ 94. Le peuplement des îles méditerranéennes et le problème de l'insularité. Banyuls-sur-Mer 1959, Paris (I96I), 49-54.

Polybe ( $3^{\mathrm{e}}$ siècle avant J.C.). Histoives, Livre I 2, 3, 7-1o (intéressant passage sur la faune de la Corse à l'époque). "Les belles lettres ", Paris I96I, 3.

Pomel A., I897. Les Ovidés. Carte géol. Algérie, monog., 33 p., $\mathrm{I}_{4} \mathrm{pl}$.

Pommerol F., I880. Le mouflon quaternaire. Ass. Franç. Avancement des Sciences. C. R. $8^{\mathrm{e}}$ Sess. Montpellier 1879 (Paris I880), 600-609, fig. 67-7 I, pl. 3 . 
Pommerol F., i 882. Recherches sur le moufon quaternaire (Ovis antiqua). Ass. Fr. Av. Sc. C. R. Io $0^{\mathrm{e}}$ Sess., Alger $188 \mathrm{I}$ (Paris I882), 525-530, fig. 68-7I.

Poplin F., i977. Paléontologie du mouton, in: Les débuts de l'élevage du Mouton. Colloque d'Ethnozootechnie, Alfort 1977 (Ethnozootechnie no 2I), Paris, 9-1o.

Poplin F., I979. Le destin de la grande faune européenne à la fin des Temps glaciaires : le changement de nature et l'appel de la domestication, in : la fin des Temps glaciaires en Europe. Coll. Intern. du C.N.R.S., no $27 I$, Bordeaux 1977, Paris, I979, 78-84.

Poplin F., 1978. Nascita e destino della caccia e della domesticazione (Origine et destinée de la chasse et de la domestication des animaux). Enciclopedia della Scienza e della Tecnica, Mondadori, Milan, 205-216.

QUiTter E., I976. Races ovines françaises. La maison rustique, Paris $2^{\mathrm{e}}$ éd., I95 p.

Reed C. A. et Schaffer W., I966. Evolutionary implications of cranial morphology in the sheep and goats (Caprini, Simpson 1945). Amer. Zool., 6, 565.

Rozoy J.-G., 1978. Les derniers chasseurs. Bull. Soc. Archéol. Champenoise, no spécial, 3 vol., I 256 p., 294 fig., 259 pl., 8I tabl.

Ryder M. L., I97I. The status of non-domestic sheep. Antiquity, 45, 220-222.

RYDER M. L., I973. The use of the skin and coat in studies of changes following domestication, in: Domestikationsforschung und Geschichte der Haustiere. Int. Symp. Budapest I97I (I973), Acad. Sc., Budapest.

Ryder M. L., I974. Wools from antiquity. Textile history, 5, 100-1 10, Pasold Research Fund Ltd.

Schaffer W. M. et ReED C. A., 1972. The co-evolution of social behaviour and cranial morphology in sheep and goats (Bovidae, Caprini). Fieldiana Zoology, 61, I, I-88, 26 fig., 33 pl.

Schaub S., I923. Neue und wenig bekannte Cavicornier von Senèze. Eclog. geol. Helvetiae, 18, 28I-295, 5 fig.

Schaub S., r932. Die Ruminantier des ungarischen Praeglacials. Eclog. geol. Helvetiae, 25 , 2, 3 I9-330.

Schaub S., i937. Ein neuer Cavicornier aus dem Oberpliocaen von Honan. Bull. Geol. Instit. Upsala, 27, 25-31, 2 fig.

Schaub S., I944. Die oberpliocaene Säugetierfauna von Senèze (Haute-Loire) und ihre verbreitungsgeschichtliche Stellung. Eclog. geol. Helvetiae, 36, 2, I943 (1944) 270-289, 8 fig.

Schaub S., I95I. Soergelia n. gen., ein Caprine aus dem thüringischen Altpleistocaen. Eclog. geol. Helvetiae, 44, 2, 375-381, 4 fig., pI. I I-I 2.

Scrres M. de, Dubreuil et Jeanjean, 1839. Recherches sur les ossemens humatiles des cavernes de Lunel-Viel, 275 p., 20 pl., I plan, Boehm et Cie, Montpellier.

Simpson G. G., 1945. The principles of classification and a classification of the Mammals. Bull. Amer. Mus. Nat. Hist., 85, $35^{\circ} \mathrm{p}$.

Stehlrn H.-G., r932. Paléontologie des couches paléolithiques, in: Dubois (A.) et SteHLiN (H.-G.). La giotte de Cotencher, station moustérienne. Mém. Soc. Pal. Suisse, 52, 33-I 78, pl. 5-9.

Teilhard de Chardin P. et Piveteau J., i93o. Les mammifères fossiles de Nihowan (Chine). Ann. Paléont., 19, 134 p., 42 fig., 23 pl.

Thenius E. et Hofer H., I960. Stammesgeschichte der Säugetieve, eine Übersicht über Tatsachen und Probleme der Evolution dev Saugetieve, 322 p., 53 fig., 2 tabl., Springer, Berlin, Göttingen, Heidelberg.

Tobien H., 1935. Über die pleistozänen und postpleistozänen Prolagusformen Korsikas und Sardiniens. Ber. Naturf. Ges. $z$. Freiburg $i$. Br., 34, 253-344, 6 fig., I pl., I 2 tabl.

Tsal kin W. I., i95I. Les Mouflons d'Europe et d'Asie (en russe). M. O. I. P., Moscou.

Türcke F. et Schmincke S., I965. Das Muffelwild, I93 p., nombreuses fig., Paul Parey, Hambourg et Berlin.

Uloth W., I976. Das Muffelwild = Ovis ammon musimon. Die Neue Brehm Bücherei, Io4 p.

Vaillant-Couturier I. et Vaillant-Couturier P., i 928. La grotte azilienne du "Trou Violet" à Montardit (Ariège). L'Anthropologie, 38, 217-243, 16 fig.

VAUFrey R., 1929. Les éléphants nains des îles méditerranéennes et la question des isthmes pléistocènes. Arch. Inst. Paléont. Humaine. Mém. 6, 220 p., 45 fig., 9 pl., Masson, Paris.

VIret J., r96r. Super-famille : Bovoidea, in: Traité de Paléontologie, sous la dir. de J. PrveTEAU, 6, Mammifèves: origine reptilienne et évolution, Masson, Paris, 1038-1084, fig. 136-166.

Zeuner F. E., 1963. A history of domesticated animals, 560 p., nombreuses fig., Hutchinson, Londres. 